\title{
Retraction Note to: Control and Automation, and Energy System Engineering
}

\author{
Tai-hoon Kim ${ }^{1}$, Hojjat Adeli ${ }^{2}$, Adrian Stoica ${ }^{3}$, and Byeong-Ho Kang ${ }^{4}$ \\ ${ }^{1}$ Hannam University, Daejeon, Korea \\ taihoonn@empas.com \\ ${ }^{2}$ The Ohio State University, Columbus, OH, USA \\ adeli.1@osu.edu. \\ ${ }^{3}$ Jet Propulsion Laboratory, Pasadena, CA, USA \\ adrian.stoicaljpl.nasa.gov \\ ${ }^{4}$ University of Tasmania, Hobart, TAS, Australia \\ byeong. kang@utas.edu. au
}

\section{Retraction Note to: \\ Chapter 8 \\ Characteristics Analysis of the Motor Block Lattice Resistor of a High Speed Train by Structure Improvement DOI: 10.1007/978-3-642-26010-0_8}

The chapter starting on page 65 of this publication has been retracted due to multiple publication

\section{Retraction Note to: \\ Chapter 38 \\ Characteristics Analysis of the Motor Block Lattice Resistor of a High Speed Train by Structure Improvement DOI: 10.1007/978-3-642-26010-0_38}

The chapter starting on page 302 of this publication has been retracted due to multiple publication.

\footnotetext{
The updated original online version for these chapters can be found at DOI: $10.1007 / 978-3-642-26010-0 \_8$

DOI: $10.1007 / 978-3-642-26010-038$

DOI: $10.1007 / 978-3-642-26010-0 \_39$

DOI: $10.1007 / 978-3-642-26010-0 \_40$ 
Retraction Note to:

Chapter 39

Estimation of Deterioration Degree in Overhead Transmission

Lines by Tension Load Analysis

DOI: 10.1007/978-3-642-26010-0_39

The chapter starting on page 312 of this publication has been retracted as it is a duplicate of the paper starting on page 86

Retraction Note to:

Chapter 40

Structure Vibration Analysis and Active Noise Control of a Power Transformer by Mobility Measurement

DOI: 10.1007/978-3-642-26010-0_40

The chapter starting on page 322 of this volume has been retracted due to multiple publication. 